\title{
Thermodynamic study of canned mince meat and fish in metal cans during $\mathbf{3 0}$ months storage
}

\author{
Vanya Gandova ${ }^{1, *}$ \\ ${ }^{1}$ Department of Analytical and Physical chemistry, University of Food technologies, 4002 Plovdiv, Bulgaria
}

\begin{abstract}
Long storage period for the meat and fish cans of 30 months was presented. The provide investigation aims to found thermodynamic stability in mackerel in tomato sauce and finely ground pork minced meat. During the storage period a decrease in $\mathrm{pH}$ in two types of cans were seen. $\mathrm{pH}$ values for mackerel in tomato sauce and for finely ground pork minced meat cans were measured between $6.2 \pm 0.19$ to $4.8 \pm 0.22$ and $5.8 \pm 0.24$ to $3.9 \pm 0.17$ in the starting to the end of storage, respectively. Determined absorption is used to calculated equilibrium constant, Gibbs free energy $(\Delta \mathrm{G})$, enthalpy $(\Delta \mathrm{H})$ and entropy $(\Delta \mathrm{S})$. For mackerel in tomato sauce $\Delta \mathrm{G}$ exhibit value $-8.33 \pm 0.07 \mathrm{~kJ} . \mathrm{mol}^{-1}$ and for finely ground pork $-6.79 \pm 0.09$ $\mathrm{kJ} . \mathrm{mol}^{-1}$. Microscopic examinations were performed and the size of the particles was determined. In measurements in mackerel in tomato sauce the sizes are between $25 \pm 0.79$ to $62 \pm 1.28 \mu \mathrm{m}$ and for finely ground pork minced meat the particle sizes are between $32 \pm 1.12$ to $89 \pm 2.48 \mu \mathrm{m}$ in the investigation period. Based on the experiment, both types of canned food are defined as thermodynamically stable after negative Gibbs energies observations, but canned fish is defined as more stable and may have a longer stored.
\end{abstract}

\section{Introduction}

The foods are protected in aluminum foil, metal cans and glass jars [1]. A shelf life of 1 year stored at a temperature $28 \pm 2 \mathrm{C}$ was investigated from authors [2]. Thermal processing for fish preservation with long-term stability for different seafood products was presented. The changes in nutrition for vitamins, colour and microbial growths were observed as results. Djenane and Roncalés [3] presented gases such as carbon monoxide (CO) appropriate to extended shelf life of fresh muscle foods in the food packaging. Another investigation with application of $\mathrm{CO}_{2}$-emitter was used for long shelf life in different types of foods, Eie et al. [4]. Their investigations make applications in cheese, butter, oats, fresh cod, ground beef, poultry meat, fresh salmon and smoked salmon. Active intelligent packaging technologies were presented for prolonging shelf life and improving the quality of food according to Han et al. [5]. They were found improvements in new characteristics of food packaging, such as mechanical strength, barrier performance and thermal stability. Investigation of the effect of different packaging methods - air, vacuum and atmosphere was ensured from authors [6]. The investigations presented the influence of different parameters (oxygen, enzymes, temperature, moisture, microorganisms, light). Zho at al. [7] supply results for different meat products preserved in their shelf life. During the storage of meat good manipulation practices were used to conserve and inhibit microbial growth. The preservation process is based on refrigeration. Using a different type of packaging, maximum shelf life can be

\footnotetext{
Corresponding author: gandova 71@abv.bg
}

achieved [8]. Different studies describing the ability of omega-3 fish oil and thermodynamic analyses were provided [9]. Adadi at al. [10] investigated $\mathrm{pH}$ in beer after fermentation and observed a decrease in $\mathrm{pH}$ during the process.

Thermodynamic conditions in the investigated system presented from three parameters Gibbs free energy, enthalpy and entropy. Gibbs free energy is connected with the equilibrium in the system, enthalpy with the thermal effects and entropy with the direction of the processes and order. Before calculated thermodynamic parameters equilibrium constant of the process between dispersed phase and continuous phase was determined. Change of concentration in different solutions from type $(\mathrm{w} / \mathrm{v})$ between 0.2 to 1.0 percent was observed [11-13]. The equilibrium constant can accept values less than 1 and greater than 1 . When the constant is positive the system can be described as thermodynamically stable but when the constant is negative the system can be described as thermodynamically unstable.

Thermodynamic data obtained with thermodynamic parameters calculations usually used to determine the moment when one colloidal system starts to destroy. Changes appeared for example with increasing size in emulsion droplets, increasing oxidation number in oils, thermal changes with destroying of colour in meat. Equilibrium constant and thermodynamic parameters Gibbs free energy $(\Delta \mathrm{G})$, enthalpy $(\Delta \mathrm{H})$ and entropy $(\Delta \mathrm{S})$ were used to predict stability in the co pigmentation between aronia and sinapic acid [14]. The interactions between strawberry anthocyanins (pigment) and rose 
petal polyphenols (co pigment) were studied to determine thermodynamic stability. Based on obtained thermodynamic parameters exothermic and thermally reversible processes were seen [15]. Investigation of oxidative stability for echium oil supplemented with SJL extract was presented from Mihaylova et al. [16]. Enthalpy of activation $\left(\Delta \mathrm{H}^{*}\right)$, entropy of activation $\left(\Delta S^{\ddagger}\right)$, and Gibbs free energy of activation $\left(\Delta G^{\star}\right)$ were calculated. Good stability after storage at three temperatures in the oil it is found.

Aspergillus carbonarius NRRL369 investigation was presented for purification of lipase with aqueous twophase system (ATPS). Changes of some thermodynamic parameters - enthalpy $(\Delta \mathrm{H})$, entropy $(\Delta \mathrm{S})$ and the free Gibbs energy $(\Delta G)$ of the system were calculated which are characteristics of the thermodynamic stability. The partition of the enzyme in the top phase is a spontaneous process connected with negative values obtained for Gibbs energy $(\Delta \mathrm{G})$. Values of the change in the enthalpy $(\Delta \mathrm{H})$ and the entropy $(\Delta \mathrm{S})$ reveal that the process was exothermic [17].

So far not found data for determination of thermodynamic parameters in cans in the literature. Stored period in investigated cans connected with some changes which can be explained with different thermodynamic parameters in beginning and end of storage.

The aim in this study is to investigate different cans at long storage periods compatible with their shelf life. Thermodynamic parameters are physicochemical characteristics. In this case they determine thermodynamic stability and can be used to predict when to start to destroy some fish or meat cans and how long they can be stored.

\section{Materials and Methods}

Mackerel in tomato sauce and finely ground pork minced meat cans were provided for investigations from local producer Compass. For preparation of solutions distilled water was used.

From each can (fish or meat) $25 \mathrm{~g}$ was taken only from the solid part of the can and mixed with $25 \mathrm{ml}$ water. A homogenizer was used for mixing in duration of $3 \mathrm{~min}$.

$\mathrm{pH}$ meter SUP-PH5011 with $\mathrm{pH}$ sensor is applied to some $\mathrm{pH}$ determinations. All measurements were provided immediately after preparation of dilutions.

CamSpec M107 UV/VIS spectrophotometer with 1 $\mathrm{cm}$ plastic cuvette path was used for determined measurements of absorption [15]. Preliminary wavelength for spectrophotometer measurements was determined at $350 \mathrm{~nm}$. For determination of absorption a series of solutions with increasing concentrations from $0.2 \%$ to $1.0 \%$ were prepared and investigated.

First equilibrium constant was determined by graphical dependence. Using eq. 1 Gibbs free energy was found.

$$
\Delta G=-R \cdot T \cdot \ln K
$$

where: $\Delta \mathrm{G}$ - Gibbs free energy; $\mathrm{K}$ - equilibrium constant; $\mathrm{R}$ - gas constant; $\mathrm{T}$ - temperature in Kelvin.

By equation (2) the enthalpy was determining:

$$
\frac{d \ln K}{d(1 / T)}=-\frac{\Delta H}{R}
$$

where: $\Delta \mathrm{H}$ - enthalpy.

The entropy $(\Delta S)$ was calculated from the equation (3):

$$
\Delta S=\frac{(\Delta H-\Delta G)}{T}
$$

where: $\Delta \mathrm{S}$ - entropy.

Microscopic investigations of cans were performed with an optical microscope (Olympus SZX7; Camera Systems Include 4.9MP Color).

Micrographs were collected by using image analysis software, at $80 \times$ magnification. The mean droplet size was reported by using micrometric scale.

The results present in this study are the mean values from three determinations. The statistical analyses were performed with one-way ANOVA to test levels of significant difference among the means, followed by Tukey's multiple comparison $(p=0.05)$, according Assaad et al. [18].

OriginPro 7.0 software for statistical presentation for linear regression analysis was applied.

\section{Results and Discussion}

Investigation of equilibrium constant in the two types of cans was performed. In the mackerel in tomato sauce cans obtained results presented numbers between $28.15 \pm 0.42$ in the beginning of measurements to $12.42 \pm 0.26$ in the end. In the finely ground pork minced meat cans observed low numbers between $15.74 \pm 0.12$ when starting measurements to $4.58 \pm 0.31$ to finish the experiment. Comparative review between equilibrium constants in two types of cans are presented on Fig. 1.

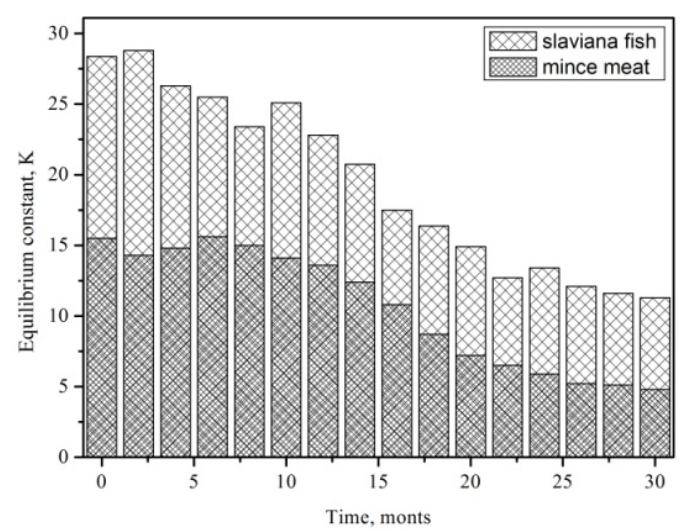

Fig. 1. Comparative review on the equilibrium constant $\mathrm{K}$ between mackerel in tomato sauce and finely ground pork minced meat cans. 
High equilibrium constant connected with a more stable system. According to the figure the fish cans are more stable compared to minced meat.

Gibbs free energy, enthalpy and entropy for fish and meat cans are presented in the Tables 1 and 2. Negative values for all parameters were obtained. For mackerel in tomato sauce cans the Gibbs free energy exhibits some highest values from $-8.33 \pm 0.07 \mathrm{~kJ} \mathrm{~mol}^{-1}$ in first months to $6.01 \pm 0.04 \mathrm{~kJ} \mathrm{~mol}^{-1}$ to last. For finely ground pork minced meat cans Gibbs free energy exhibits values between $-6.79 \pm 0.09 \mathrm{~kJ} \mathrm{~mol}^{-1}$ to $-3.89 \pm 0.01 \mathrm{~kJ} \mathrm{~mol}^{-1}$ from start to end of the investigated period.

Table 1. Gibbs free energy, enthalpy and entropy values calculated for mackerel in tomato sauce.

\begin{tabular}{|c|c|c|c|}
\hline Months & $\boldsymbol{\Delta G}$ & $\boldsymbol{\Delta H}$ & $\boldsymbol{\Delta S}$ \\
\hline 0 & $-8.29 \pm 0.07$ & $-20.94 \pm 0.03$ & $-0.04 \pm 0.02$ \\
\hline 2 & $-8.33 \pm 0.07$ & $-20.96 \pm 0.03$ & $-0.04 \pm 0.02$ \\
\hline 4 & $-8.10 \pm 0.10$ & $-20.86 \pm 0.08$ & $-0.04 \pm 0.01$ \\
\hline 6 & $-8.02 \pm 0.11$ & $-20.83 \pm 0.03$ & $-0.04 \pm 0.01$ \\
\hline 8 & $-7.81 \pm 0.14$ & $-20.74 \pm 0.11$ & $-0.04 \pm 0.01$ \\
\hline 10 & $-7.99 \pm 0.08$ & $-20.81 \pm 0.10$ & $-0.04 \pm 0.01$ \\
\hline 12 & $-7.75 \pm 0.04$ & $-20.71 \pm 0.12$ & $-0.04 \pm 0.05$ \\
\hline 14 & $-7.51 \pm 0.04$ & $-20.61 \pm 0.07$ & $-0.04 \pm 0.01$ \\
\hline 16 & $-7.09 \pm 0.07$ & $-20.42 \pm 0.01$ & $-0.04 \pm 0.04$ \\
\hline 18 & $-6.93 \pm 0.07$ & $-20.35 \pm 0.05$ & $-0.05 \pm 0.03$ \\
\hline 20 & $-6.69 \pm 0.11$ & $-20.25 \pm 0.01$ & $-0.05 \pm 0.02$ \\
\hline 22 & $-6.30 \pm 0.13$ & $-20.08 \pm 0.10$ & $-0.05 \pm 0.02$ \\
\hline 24 & $-6.43 \pm 0.02$ & $-20.14 \pm 0.12$ & $-0.05 \pm 0.02$ \\
\hline 26 & $-6.18 \pm 0.09$ & $-20.03 \pm 0.13$ & $-0.05 \pm 0.02$ \\
\hline 28 & $-6.07 \pm 0.07$ & $-19.98 \pm 0.09$ & $-0.05 \pm 0.01$ \\
\hline 30 & $-6.01 \pm 0.04$ & $-19.95 \pm 0.11$ & $-0.05 \pm 0.01$ \\
\hline
\end{tabular}

Table 2. Gibbs free energy, enthalpy and entropy values calculated for finely ground pork minced meat.

\begin{tabular}{|c|c|c|c|}
\hline Months & $\boldsymbol{\Delta G}$ & $\boldsymbol{\Delta H}$ & $\boldsymbol{\Delta} \mathbf{S}$ \\
\hline 0 & $-6.79 \pm 0.09$ & $-20.29 \pm 0.22$ & $-0.05 \pm 0.01$ \\
\hline 2 & $-6.59 \pm 0.11$ & $-20.21 \pm 0.13$ & $-0.05 \pm 0.08$ \\
\hline 4 & $-6.68 \pm 0.04$ & $-20.24 \pm 0.11$ & $-0.05 \pm 0.07$ \\
\hline 6 & $-6.81 \pm 0.02$ & $-20.30 \pm 0.11$ & $-0.05 \pm 0.02$ \\
\hline 8 & $-6.71 \pm 0.02$ & $-20.26 \pm 0.08$ & $-0.05 \pm 0.02$ \\
\hline 10 & $-6.56 \pm 0.03$ & $-20.19 \pm 0.10$ & $-0.05 \pm 0.02$ \\
\hline 12 & $-6.47 \pm 0.04$ & $-20.15 \pm 0.11$ & $-0.06 \pm 0.01$ \\
\hline 14 & $-6.24 \pm 0.08$ & $-20.05 \pm 0.11$ & $-0.06 \pm 0.01$ \\
\hline 16 & $-5.90 \pm 0.02$ & $-19.90 \pm 0.09$ & $-0.06 \pm 0.01$ \\
\hline 18 & $-5.36 \pm 0.07$ & $-19.67 \pm 0.08$ & $-0.05 \pm 0.01$ \\
\hline 20 & $-4.89 \pm 0.07$ & $-19.47 \pm 0.02$ & $-0.05 \pm 0.01$ \\
\hline 22 & $-4.64 \pm 0.04$ & $-19.36 \pm 0.21$ & $-0.06 \pm 0.04$ \\
\hline 24 & $-4.40 \pm 0.05$ & $-19.25 \pm 0.17$ & $-0.06 \pm 0.04$ \\
\hline 26 & $-4.09 \pm 0.01$ & $-19.12 \pm 0.14$ & $-0.05 \pm 0.07$ \\
\hline 28 & $-4.04 \pm 0.01$ & $-19.10 \pm 0.12$ & $-0.06 \pm 0.05$ \\
\hline 30 & $-3.89 \pm 0.01$ & $-19.03 \pm 0.12$ & $-0.06 \pm 0.05$ \\
\hline
\end{tabular}

The Gibbs energy and equilibrium constant exhibit high values in the mackerel in tomato sauce cans compared with finely ground pork mincemeat cans and determine these cans with greater stability. Decreasing of Gibbs free energy and equilibrium constant during the storage period to the last months probably connected with destroying processes.
Negative enthalpy was observed in the two cans. These results determined processes as exothermic and the energy of the products is less than the energy of the reactants. Thermodynamic connection between Gibbs free energy and enthalpy presented negative energy with positive entropy. According to calculations in two types of cans small negative entropy was observed. This negative sign means that there is an observed disorder in the system. When it appears less disordered, energy must be used. A spontaneous process of destruction was observed. Dependence between Gibbs free energy and time in months is presented on Fig. 2. The figure shows that with the increasing time of storage the Gibbs free energy leads to small values. This is connected with the process occurring in the system. These processes are connected with breakdown of protein and fats.

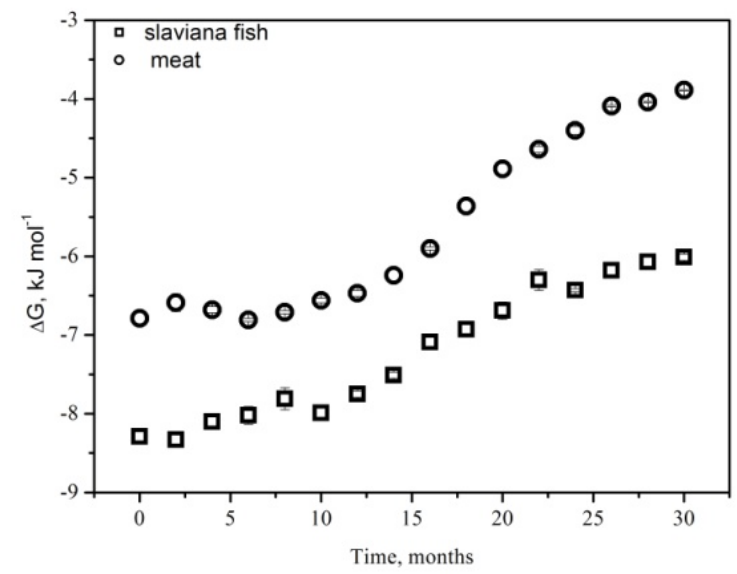

Fig. 2. Comparative review on the Gibbs free energy between mackerel in tomato sauce and finely ground pork minced meat cans.

During the storage period the $\mathrm{pH}$ values were measured. A decrease in $\mathrm{pH}$ in two types of cans were seen. The foods are divided into two parts: a high-acid food exhibits a $\mathrm{pH}$ of less than 4.6 and a low-acid food exhibits a pH higher than 4.6. According these classifications, the mackerel in tomato sauce and finely ground pork minced meat are classified as high-acid foods. $\mathrm{pH}$ values for mackerel in tomato sauce were measured at $6.2 \pm 0.19$ in the starting investigations to $4.8 \pm 0.22$ in the last months. For finely ground pork minced meat cans $\mathrm{pH}$ was determined between $5.8 \pm 0.24$ in the beginning to $3.9 \pm 0.17$ to the end. Supplements are the greater part of the requirement as minerals and vitamins. According to the processes in the cans, drops appear that they increase values. Observed colloidal interactions, appeared solid parts in the cans and $\mathrm{pH}$ decrease connected with these processes.

Mackerel in tomato sauce and finely ground pork minced meat cans were extra investigated with microscopic investigations. Microscopic observations of them were presented to Figs. 3 and 4. Small droplets can be seen on Fig. 3 in the mackerel in tomato sauce cans compared with another from Fig. 4 on finely ground pork minced meat. These results are observed after the first measurements. The small sizes of droplets connected to the stability of the system and small Gibbs 
free energy. During the storage period as results of the process have taken place the droplets increase their size. For example, in mackerel in tomato sauce cans observed particles with sizes between $25 \pm 0.79$ to $62 \pm 1.28 \mu \mathrm{m}$ from start to end of measurements during the storage period. Compared with finely ground pork minced meat cans fish cans exhibit the smallest particles. In finely ground pork minced meat cans particle sizes were determined at $32 \pm 1.12$ to $89 \pm 2.48 \mu \mathrm{m}$ from start to end. Increasing the particle size connected with colloidal interactions between proteins, fats and water in them. The hydrophobic interactions of the proteins lead to bad adsorption in emulsions.

Diffusion processes, destruction and protein loss are related to thermal changes. Addition of a high amount of amino acid protects canned fish products against this process [19].

Good correlation between particle sizes after optical microscope observation and Gibbs free energy was established. Small particles in mackerel in tomato sauce are connected with appropriate small Gibbs energies compared with particle sizes and Gibbs energies at finely ground pork minced meat can [20].

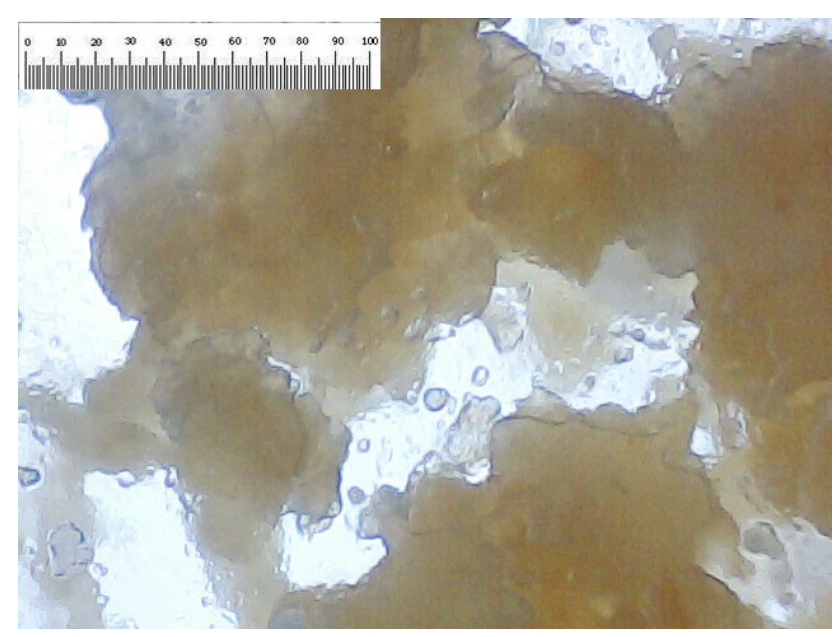

Fig. 3. Micrograph of mackerel in tomato sauce can.

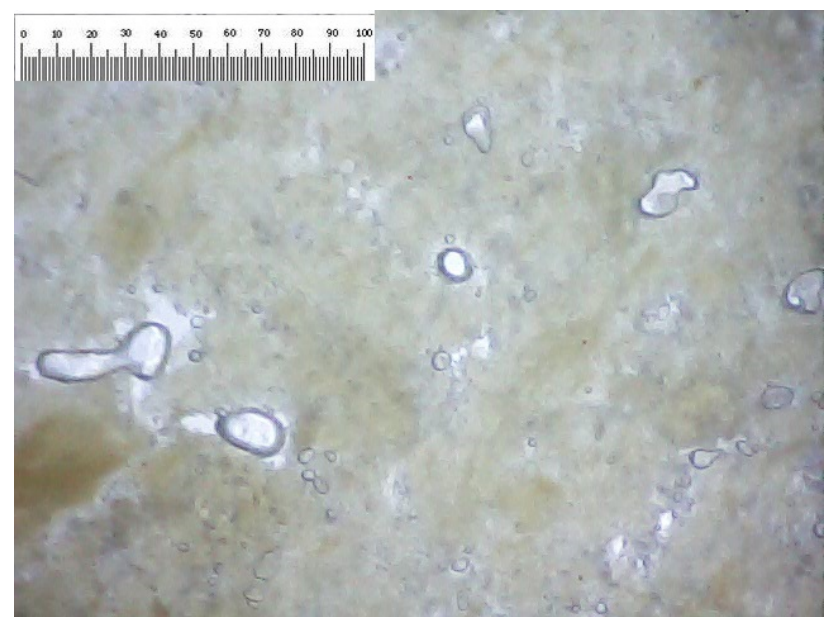

Fig. 4. Micrograph of finely ground pork minced meat can.

\section{Conclusion}

Two types of cans mackerel in tomato sauce and finely ground pork minced meat were investigated after observed changes of $\mathrm{pH}$, absorption and particle sizes in them. Long storage period was provided compatible with their shelf life. Based on the results of the experiment it is observed a decrease of $\mathrm{pH}$, an increase of of absorption connected with decreasing of equilibrium constant and increasing of particle sizes during the storage. After providing experiment thermodynamic parameters equilibrium constant, Gibbs free energy $(\Delta \mathrm{G})$, enthalpy $(\Delta \mathrm{H})$ and entropy $(\Delta \mathrm{S})$ were calculated. Mainly Gibbs energy is related to the thermodynamic stability of the studied systems. In this case $\Delta \mathrm{G}$ exhibits values for mackerel in tomato sauce $-8.33 \pm 0.07 \mathrm{~kJ}^{\mathrm{mol}}{ }^{-1}$ and for finely ground pork minced meat $-6.79 \pm 0.09$ $\mathrm{kJ} \cdot \mathrm{mol}^{-1}$. After the analyses and calculations, it can be concluded that fish cans exhibit higher thermodynamic stability.

Acknowledgements: The author gratefully acknowledges the financial support by Project 04/20-H, University of Food Technologies, Plovdiv, Bulgaria.

\section{References}

1. Y. Shan, Canned citrus processing. Techniques, equipment, and food safety (Academic Press, Elsevier, Amsterdam - Boston - Heidelberg London - New York - Oxford - Paris - San Diego San Francisco - Singapore -Sydney - Tokyo, 2016)

2. J. Bindu, A. Mallick, T. Gopal, Fish. Technol. 130, 33 (2003)

3. D. Djenane, P. Roncalés, Foods 7, 12 (2018)

4. T. Eie, H. Larsen, O. Sorheim, M. Pettersen, A. Hansen, K. Naterstad, M. Mielnik, Ital. J. Food Sci. 19, 127 (2007)

5. J. Han, L. Garcia, J. Qian, X. Yang, Compr. Rev. Food Sci. Food Saf. 17, 860 (2018)

6. X. Zhang, W. Huang, J. Xie, Foods. 8, 325 (2019)

7. G. Zho, X. Hu, Y. Liu, Meat Sci. 86, 119 (2010)

8. R. Mathew, D. Jaganathan, Glob. J. Biol. Agric. Heal. Sci. 6, 32 (2017)

9. D. Loiselle, J. Han, E. Goo, B. Chapman, C. Barclay, A. Hickey, A. Taberner, J. Gener. Physiol. 148, 183 (2016)

10. P. Adadi, E. Kovaleva, T. Glukhareva, S. A. Shatunova, A. Petrov, Agronom. Res. 15, 1831 (2017)

11. N. Singh, D. Prazad, S. Kumar, J. Amer. Oil Chem. Soc. 70, 69 (1993)

12. Y. Bayrak, Colloids Surf. Physicochem. Eng. Asp. 247, 99 (2004)

13. O. Zheng, J. Zhao, H. Yan, S. Gao, J. Coll. Interf. Sci. 310, 331 (2007) 
14. I. Petrova, V. Gandova, IOP Conf. Series: Mater. Sci. Engin. 618, 012097 (2019)

15. I. Petrova, K. Mihalev, V. Shikov, V. Gandova, E3S Web of Conf. 207, 01020 (2020)

16. D. Mihaylova, V. Gandova, I. Deseva, S. Tschuikowa, S. Schalow, G. Westphal, Eur. J. Lipid Sci. Technol., 122, e2000118 (2020)

17. H. Panajotova, H. Strinska, V. Gandova, V. Dobreva, B. Zhekova, G. Dobrev, Bulg. Chem. Commun. 49, 130 (2017)

18. H. Assaad, L. Zhou, R. J. Carroll, G. Wu, Springer Plus. 3, 474 (2014)

19. A. Bender, J. Food Technol. 7, 239 (1972)

20. E. Figueiredo, J. Arêasb, E. Arêas, J. Braz. Chem. Soc. 19, 1336 (2008) 